\title{
TrkB-Shc Signaling Protects against Hippocampal Injury Following Status Epilepticus
}

\author{
CPang Zhong Huang, ${ }^{1}$ Xiao-Ping He, ${ }^{1}$ Kamesh Krishnamurthy, ${ }^{1}$ and James 0. McNamara ${ }^{1,2,3}$ \\ Departments of ${ }^{1}$ Neurobiology, ${ }^{2}$ Medicine (Neurology), and ${ }^{3}$ Pharmacology and Molecular Cancer Biology, Duke University Medical Center, Durham, \\ North Carolina 27710
}

Temporal lobe epilepsy (TLE) is a common and commonly devastating form of human epilepsy for which only symptomatic therapy is available. One cause of TLE is an episode of de novo prolonged seizures [status epilepticus (SE)]. Understanding the molecular signaling mechanisms by which SE transforms a brain from normal to epileptic may reveal novel targets for preventive and disease-modifying therapies. SE-induced activation of the BDNF receptor tyrosine kinase, TrkB, is one signaling pathway by which SE induces TLE. Although activation of TrkB signaling promotes development of epilepsy in this context, it also reduces SE-induced neuronal death. This led us to hypothesize that distinct signaling pathways downstream of TrkB mediate the desirable (neuroprotective) and undesirable (epileptogenesis) consequences. We subsequently demonstrated that TrkB-mediated activation of phospholipase $\mathrm{C} \gamma 1$ is required for epileptogenesis. Here we tested the hypothesis that the TrkB-Shc-Akt signaling pathway mediates the neuroprotective consequences of TrkB activation following SE. We studied measures of molecular signaling and cell death in a model of SE in mice of both sexes, including wild-type and $T r k B^{S h c / S h c}$ mutant mice in which a point mutation (Y515F) of TrkB prevents the binding of Shc to activated TrkB kinase. Genetic disruption of TrkB-Shc signaling had no effect on severity of SE yet partially inhibited activation of the prosurvival adaptor protein Akt. Importantly, genetic disruption of TrkB-Shc signaling exacerbated hippocampal neuronal death induced by SE. We conclude that therapies targeting TrkB signaling for preventing epilepsy should spare TrkB-Shc-Akt signaling and thereby preserve the neuroprotective benefits.

Key words: hippocampus; neuroprotection; Shc; status epilepticus; temporal lobe epilepsy; TrkB

Significance Statement

Temporal lobe epilepsy (TLE) is a common and devastating form of human epilepsy that lacks preventive therapies. Understanding the molecular signaling mechanisms underlying the development of TLE may identify novel therapeutic targets. BDNF signaling thru TrkB receptor tyrosine kinase is one molecular mechanism promoting TLE. We previously discovered that TrkBmediated activation of phospholipase $\mathrm{C} \gamma 1$ promotes epileptogenesis. Here we reveal that TrkB-mediated activation of Akt protects against hippocampal neuronal death in vivo following status epilepticus. These findings strengthen the evidence that desirable and undesirable consequences of status epilepticus-induced TrkB activation are mediated by distinct signaling pathways downstream of this receptor. These results provide a strong rationale for a novel therapeutic strategy selectively targeting individual signaling pathways downstream of TrkB for preventing epilepsy.

\section{Introduction}

Temporal lobe epilepsy (TLE) is a common and commonly devastating form of human epilepsy because of its suboptimal re-

\footnotetext{
Received Nov. 16, 2018; revised Feb. 20, 2019; accepted March 23, 2019.

Author contributions:Y.Z.H., X.-P.H., and J.0.M. designed research; Y.Z.H., X.-P.H., and K.K. performed research;

Y.Z.H., X.-P.H., K.K., and J.O.M. analyzed data; Y.Z.H., K.K., and J.O.M. wrote the first draft of the paper; Y.Z.H.,

X.-P.H., K.K., and J.O.M. edited the paper; Y.Z.H., K.K., and J.O.M. wrote the paper.

This work was supported by a Grant from NINDS 056217 (J.0.M.), by the CURE Taking Flight Award to Y.Z.H, and by a predoctoral research fellowship from the American Epilepsy Society to K.K.

The authors declare no competing financial interests.

Correspondence should be addressed to James 0. McNamara at jmc@neuro.duke.edu.

https://doi.org/10.1523/JNEUROSCI.2939-18.2019

Copyright $@ 2019$ the authors
}

sponsiveness to antiseizure medications and frequent psychiatric comorbidities (French, 2007). Both clinical and preclinical evidence implicate an episode of prolonged seizure activity [status epilepticus (SE)] as one cause of TLE in humans. Retrospective and longitudinal clinical studies identified an episode of SE as a risk factor for development of TLE (Annegers et al., 1987, French et al., 1993, Tsai et al., 2009; Hesdorffer et al., 2016). Experimentally inducing SE by diverse methods is sufficient to induce late onset TLE in species ranging from rodents to nonhuman primates (Pitkänen, 2010).

Understanding the molecular signaling mechanisms by which SE transforms a brain from normal to epileptic may reveal novel 
targets for preventive and disease-modifying therapies for TLE. Work by multiple investigators including ourselves implicates the receptor tyrosine kinase, $\operatorname{TrkB}$, and its canonical ligand, BDNF, as one signaling pathway by which SE induces development of TLE (McNamara and Scharfman, 2012). The binding of BDNF to the ectodomain of TrkB leads to phosphorylation of tyrosines 515 (Y515) and 816 (Y816) in the cytoplasmic domain of TrkB. Phosphorylation of residue Y515 promotes association of TrkB with the adaptor protein Shc and activation of PI3-kinase (PI3K)/Akt, MAPK signaling pathways whereas phosphorylation of residue Y816 promotes association of phospholipase $\mathrm{C} \gamma 1$ (PLC $\gamma 1$ ) and signaling mediated by IP3 and DAG (Reichardt, 2006).

Transient inhibition of TrkB kinase, initiated following SE, prevented SE-induced TLE in a mouse model (Liu et al., 2013). Although this consequence of SE-induced excessive activation of TrkB is clearly undesirable, activation of TrkB in this context also had a desirable consequence, namely protecting neurons from SE-induced death (Gu et al., 2015). This led us to hypothesize that perhaps the desirable and undesirable consequences of SEinduced activation of TrkB were mediated by distinct downstream signaling pathways. We subsequently discovered that SE-induced activation of TrkB-PLC $\gamma 1$ signaling mediates undesirable consequences, namely epilepsy and anxiety-like behavior (Gu et al., 2015). Here we used genetic and molecular methods to test the hypothesis that the TrkB-Shc signaling pathway mediates the neuroprotective effects of SE-induced TrkB activation.

\section{Materials and Methods}

Animals. TrkB $B^{s h c / s h c}$ mutant mice were generated as described previously (Minichiello et al., 1998) and had been back-crossed with C57BL/6 (Charles River Laboratories) for six generations. In brief, PCR-aided mutagenesis was used to introduce a tyrosine to phenylalanine mutation of tyrosine 515 of TrkB receptor. Y515F mutation disrupted Shc adaptor binding to this nonphosphorylated F515, thereby abolishing Shc protein-mediated downstream PI3K-Akt and MAPK signaling. In the present study, the genotype of each animal was assessed twice using PCR of genomic DNA isolated from the tail both before experimentation and after being killed. Adult male and female WT $(+/+, n=$ $28)$ and homozyogous $\operatorname{Trk} B^{\text {shc/shc }}$ mutant $(n=29)$ mice were used in these experiments.

Surgery and amygdala kainic acid microinfusion. Adult male and female mice $(20-30 \mathrm{~g})$ were anesthetized and placed in a stereotaxic frame. A 3.6 $\mathrm{mm}$ guide cannula (Plastics One) was inserted above the right amygdala (coordinates from bregma: $\mathrm{AP}=-1.0 \mathrm{~mm}$; $\mathrm{ML}=2.9 \mathrm{~mm}$; Gu et al., 2015). A bipolar electrode was placed into the left dorsal hippocampus (coordinates from bregma: $\mathrm{AP}=-2.0 \mathrm{~mm}, \mathrm{ML}=-1.6 \mathrm{~mm}, \mathrm{DV}=$ $-1.7 \mathrm{~mm}$ below dura). After a postoperative recovery of at least $5 \mathrm{~d}$, animals were gently restrained and an infusion cannula (Plastics One) was inserted into the right amygdala through the guide cannula to a depth of $4.6 \mathrm{~mm}$ below the dura. Either kainic acid (KA; $0.3 \mu \mathrm{g}$ in $0.5 \mu \mathrm{l}$ PBS) or vehicle $(0.5 \mu \mathrm{l}$ PBS $)$ was infused into the right amygdala at the rate of $0.11 \mu \mathrm{l} / \mathrm{min}$. The infusion cannula was left in the right amygdala for 2 additional minutes to reduce reflux.

Continuous hippocampal EEG telemetry (Grass Instrument) and time-locked video-monitoring were performed using Harmonie software (Stellate Systems). Monitoring started $15 \mathrm{~min}$ before amygdala KA infusion for recording baseline EEG and behavioral activity. EEG patterns consistent with electrographic SE included any of the following: (1) discrete electrographic seizures; (2) waxing and waning epileptiform activity; (3) continuous, high-amplitude, rapid spiking; and (4) periodic epileptiform discharges on a relatively flat background as described previously (Walton and Treiman, 1988; Treiman et al., 1990). Behavioral seizures were classified according to a modification of Racine scale for mice (Racine, 1972; Borges et al., 2003): 0, normal activity; 1, arrest and rigid posture; 2 , head nodding; 3 , partial body clonus (unilateral forelimb clonus); 4 , rearing with bilateral forelimbs clonus; 5 , rearing and falling (loss of postural control); and 6, tonic-clonic seizures with running and/or jumping. The duration of both behavioral and electrographic SE was determined by analyses of video and EEG data by trained observers blinded to treatment of mice.

Quantitative analysis of EEG energy content. Quantitative analysis of EEG energy content was performed using the method described previously (Lehmkuhle et al., 2009; Gu et al., 2015). A custom MATLAB (MathWorks) script calculated the running power in the $20-50 \mathrm{~Hz}$ band at a $1 \mathrm{~s}$ resolution and smoothed the resulting time-series using a $5 \mathrm{~min}$ moving average filter. The values of the smoothed gamma power time series during SE were averaged and normalized to the average of the baseline to give ratios representing the gross power increase during SE.

Western blotting. Animal were anesthetized with isoflurane and decapitated. Hippocampi ipsilateral to infusion site were rapidly dissected on ice and quickly frozen in liquid nitrogen. The hippocampi were homogenized in modified RIPA buffer containing the following (in mM: 50 Tris- $\mathrm{HCl}, \mathrm{pH} 7.4,150 \mathrm{NaCl}, 1 \% \mathrm{NP} 40,0.25 \%$ sodium deoxycholate, 5 EDTA, 3 sodium vanadate, 1 PMSF, and complete Mini protease inhibitor tablet (Roche; EDTA-free, 1 tablet/10 ml); following centrifugation at $15,700 \times g$, the supernatant was referred to as cell lysate and used for subsequent experiments. Proteins $(10-20 \mu \mathrm{g})$ were resolved by SDSPAGE. After transfer to nitrocellulose membrane, the blots were incubated overnight at $4^{\circ} \mathrm{C}$ with primary antibodies followed by incubation with secondary antibodies $(1: 10,000)$ for $1 \mathrm{~h}$ at room temperature. The antibodies were purchased from Cell Signaling Technology and the dilutions used in this study were as follow: p-Akt (\#9271; 1:1000), p-Erk (\#9101; 1:1000), Akt (\#9272; 1:1000), and Erk1/2 (\#9102; 1:1000). Shown are representative results of immunoblotting from at least two independent experiments. The immunoreactivity of individual bands on Western blots was measured by ImageJ software (National Institutes of Health). The ratio of p-Akt/Akt and p-Erk/Erk1/2 are shown as percentage of control.

Fluoro-Jade Cstaining. Mice were killed $24 \mathrm{~h}$ after infusion of vehicle or KA and perfused with PBS containing heparin (1 U/ml) followed by $4 \%$ paraformaldehyde in PBS buffer. Brains were removed, cryoprotected, frozen by slow immersion in isopentane chilled in dry ice, and sectioned. Serial $40 \mu \mathrm{m}$ coronal sections were cut through the forebrain spanning the entire hippocampus. Sections were subjected to Fluoro-Jade C (FJC; Millipore) staining as previously described (Gu et al., 2015). Stained sections were examined using a Zeiss AX10 microscopy system equipped with a $10 \times$ objective len and fluorescein filter (excitation: $485 \mathrm{~nm}$; emission: $525 \mathrm{~nm}$ ). The number of FJC-positive cells was counted in two adjacent sections from rostral (AP from bregma: -1.34 to $-1.46 \mathrm{~mm}$ ), middle (AP from bregma: $-1.82 \mathrm{~mm}$ ), and caudal (AP from bregma: -2.46 to $-2.92 \mathrm{~mm}$ ) levels of hippocampus ipsilateral to infusion site by an observer blinded to genotype using ImageJ software in a $260 \times 260$ $\mu \mathrm{m}$ field within the CA3 subfield as previously described ( $\mathrm{Gu}$ et al., 2015).

Experimental design and statistical analysis. The number of animals used in this study was determined based on the similar experiments and the observed effect sizes reported in our previous studies (Liu et al., 2013; $\mathrm{Gu}$ et al., 2015). Male and female mice were used in each of the experiments. Data analyses were conducted by investigators who were blinded to the experimental design and experimental procedures. Statistical analysis was performed with Prism 7 software (GraphPad Software). Detailed information regarding sampling and normalization is described in the figure legends. Unless otherwise noted, all values in the figures are presented as mean \pm SEM. Unless otherwise noted, comparisons between two groups were analyzed using unpaired Student's $t$ tests, whereas multigroup comparisons were analyzed using one-way ANOVA followed by Dunnett's post hoc tests. A $p<0.05$ was considered significant.

\section{Results}

Lack of antiseizure effect of genetic inactivation of TrkBShc signaling

Our previous work demonstrated that either genetic or pharmacologic disruption of TrkB-mediated activation of PLC $\gamma 1$ inhib- 
A $\quad$ WT Mice

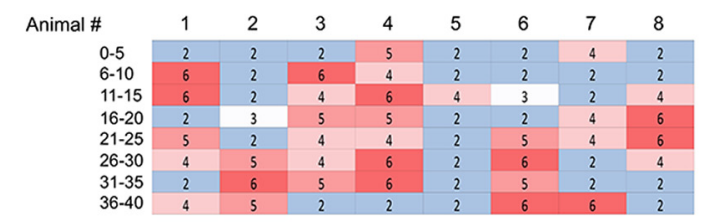

B

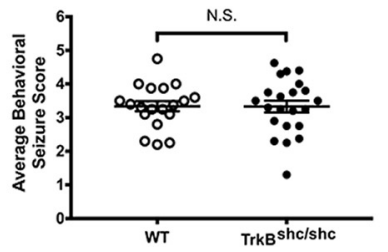

C

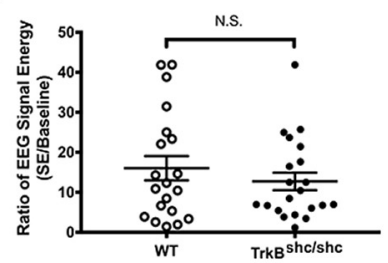

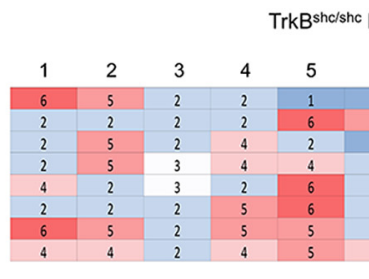

D

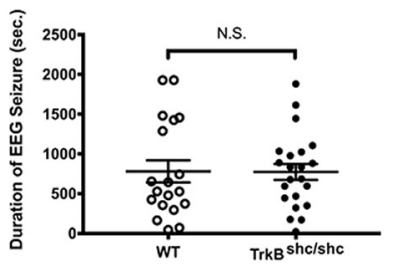

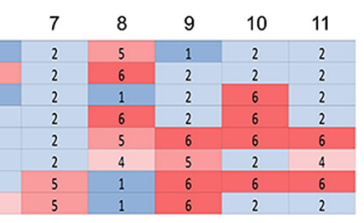

E

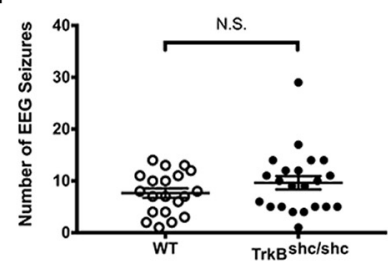

Figure 1. Infusion of KA into amygdala induces SE of similar severity in WT and $T r k B^{\text {shc/shc }}$ mice. A, Representative heat maps showing similar behavioral seizure severity during 40 min of SE in WT and $T r k B^{S h c / S h c}$ mice. Shown are the maximum seizure scores measured every $5 \mathrm{~min}$. Each row represents a 5 min epoch of SE; each column represents an individual mouse. Shades of blue indicate milder behavioral seizure scores, whereas shades of red indicate more severe seizure scores. $B$, Average behavioral seizure scores during 40 min of SE. No significant (N.S.) difference was found between WT $(n=20)$ and TrkB $B^{\text {Shc/Shc }}(n=22)$ mutant mice. C, EEG signal energy in the $20-50 \mathrm{~Hz}$ band during $40 \mathrm{~min}$ of SE. No significant differences were found between WT ( $\left.n=20\right)$ and $\operatorname{TrkB}^{\text {Shc Shc }}(n=22)$ mutant mice. $\boldsymbol{D}, \boldsymbol{E}$, Duration of EEG seizure and number of EEG seizures detected during SE. Consistent with signal energy analysis, no significant differences were found between WT and TrkB ${ }^{\text {Sh/Shc }}$ mutant mice. Data are presented as mean \pm SEM and analyzed by unpaired Student's $t$ test.

its seizures evoked by infusion of KA into amygdala ( $\mathrm{Gu}$ et al., 2015). Whether disruption of TrkB-mediated activation of Shc signaling had similar effects is unknown. To address this question, we examined behavioral and electrographic seizures evoked by intra-amygdala KA (IAK) in WT and Trk $B^{S h c / S h c}$ mice in which a phenylalanine is substituted for tyrosine at residue 515 of TrkB, thereby disrupting the binding of Shc adaptor protein to TrkB and its mediated MAPK and Akt signaling (Atwal et al., 2000). Infusion of KA induced prolonged convulsive motor seizures of similar severity in WT control mice $(n=20)$ and $\operatorname{TrkB}{ }^{S h c / S h c}(n=$ 22) mice as quantified by behavioral seizure scores (WT: $3.3 \pm$ $0.15, \operatorname{Trk} B^{S h c / S h c}: 3.3 \pm 0.18 ; p=0.9, \mathrm{df}=39$, unpaired $t$ test; Fig. $1 A, B)$. Likewise no significant differences of electrographic seizure measures were detected with respect to EEG power (WT: $16.02 \pm 3.0, \operatorname{Trk} B^{S h c / S h c}: 12.7 \pm 2.2 ; p=0.4, \mathrm{df}=39$, unpaired $t$ test; Fig. $1 C$ ) or duration (WT: $779.7 \pm 139.3 \mathrm{~s}$, TrkB ${ }^{\text {Shc/Shc }}$ : $773.9 \pm 100.3 \mathrm{~s} ; p=0.9, \mathrm{df}=39$, unpaired $t$ test; Fig. $1 D)$ or number (WT controls: $7.7 \pm 0.9, \operatorname{TrkB}^{\text {Shc/Shc }}: 9.6 \pm 1.3 ; p=0.2$, $\mathrm{df}=40$, unpaired $t$ test; Fig. $1 E$ ) of electrographic seizures. Thus, genetically uncoupling TrkB from Shc adaptor protein does not modify the extent of seizures evoked by infusion of KA into amygdala.

\section{SE-induced activation of Akt dependent on TrkB- Shc signaling}

TrkB can activate Erk and Akt signaling by a Shc-dependent mechanism in heterologous cells and cortical neurons in vitro (Reichardt, 2006). SE induced by diverse mechanisms can activate Erk and Akt as assessed in cortical lysates ex vivo (Berkeley et al., 2002; Henshall et al., 2002; Kang et al., 2004). These findings led us to ask: (1) Does SE induced by infusion of KA into amygdala activate Erk and Akt signaling and, if so, in what temporal pattern? (2) Does TrkB-Shc signaling contribute to the SEinduced activation of Erk and Akt? To assess activation of Erk and Akt, we used phosphorylation of Akt and Erk1/2 measured in hippocampal lysates as surrogates. Using mice undergoing infusion of vehicle into amygdala as controls, we examined the effects of KA-SE in animals killed at varying time points following its induction (Fig. 2A-C). KA-SE induced activation of both Erk and Akt but with striking differences in the time course of activation.
Activation of Erk was evident immediately following termination of SE with significant increases detected at $6 \mathrm{~h}$ followed by return to control levels by $24 \mathrm{~h}$ (One-way ANOVA, $\mathrm{F}=6.276, \mathrm{df}=27$, $p=0.0009$; Dunnett's post-hoc test, $p<0.001$ for Con compared with Post-KA-SE 0 hr, $p<0.05$ for Con compared with PostKA-SE $6 \mathrm{~h}$ ) (Fig. $2 \mathrm{~A}, \mathrm{C}$ ). In contrast, a trend to increased activation of Akt was detected at $6 \mathrm{~h}$ with a peak at $24 \mathrm{~h}$ followed by return to control levels by 48 and $72 \mathrm{~h}$ (One-way ANOVA, $\mathrm{F}=$ 3.249, $\mathrm{df}=27, p=0.024$; Dunnett's post-hoc test, $p<0.01$ for Con compared with Post-KA-SE 24 h) (Fig. 2A,B). The different temporal pattern of Akt and Erk activation suggests that they are activated by distinct mechanisms following SE.

To determine whether TrkB-Shc signaling contributed to KA-SE induced activation of Erk and/or Akt, we selected the $6 \mathrm{~h}$ interval following SE because of increases in both p-Erk $1 / 2$ and p-Akt at this time point. Importantly, no significant differences between WT and TrkB ${ }^{S h c / S h c}$ were detected in either Erk or Akt signaling under basal conditions (Fig. $3 A-C$ ). Comparison of Akt activation following SE evoked in WT and Trk $B^{S h c / S h c}$ mice revealed a significant reduction in $T r k B^{S h c / S h c}$ mice as evident in decreased p-Akt immunoreactivity (Fig. 3D,E; WT: $100 \pm$ $10.9 \%, n=9 ; \operatorname{Trk} B^{S h c / S h c}: 67 \pm 9.9 \%, n=12, p=0.037, \mathrm{df}=19$, unpaired $t$ test). By contrast, no differences of p-Erk1/2 immunoreactivity were detected between WT and $\operatorname{TrkB} B^{S h c / S h c}$ (Fig. $3 D, F$; WT: $100 \pm 14.2 \%, n=9 ; \operatorname{TrkB}^{\text {Shc/Shc }}: 94 \pm 14.5 \%, n=12$, $p=0.86, \mathrm{df}=19$, unpaired $t$ test). In sum, SE evokes increases of both Erk and Akt signaling but with striking differences in the temporal pattern. Moreover, assessment of animals killed $6 \mathrm{~h}$ after SE demonstrates that TrkB-Shc signaling contributes to SEinduced activation of Akt but not Erk signaling.

\section{Enhanced hippocampal neuronal death following SE in TrkB Shc/Shc}

Activation of Akt protects against neuronal death induced by KA-mediated excitotoxicity in vitro (Kim et al., 2002) and hippocampal neuronal injury induced by SE in vivo (Henshall et al., 2002). The reduction of SE-induced Akt activation in $\operatorname{TrkB} B^{S h c / S h c}$ compared with WT mice led us to hypothesize that cell death would be enhanced in $\operatorname{Trk} B^{S h c / S h c}$ mice. To test this hypothesis, we used FJC staining of hippocampal sections from mice killed $24 \mathrm{~h}$ 

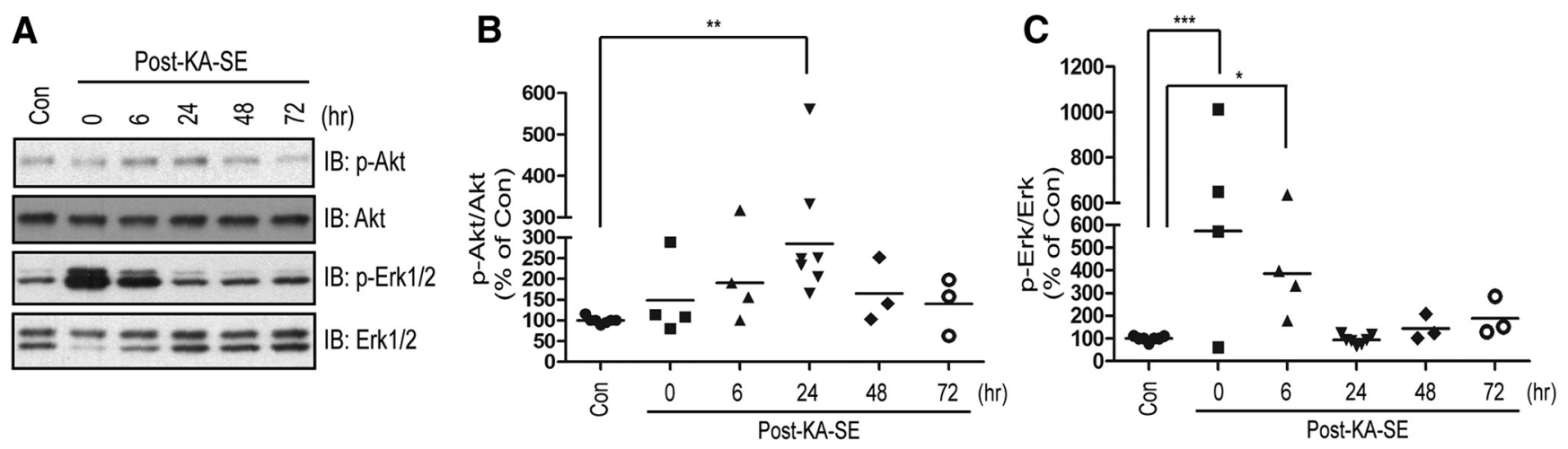

Figure 2. KA-SE induces the activation of Akt and Erk in a time-dependent manner. $A$, Time-dependent increases of phosphorylation of Akt and Erk, surrogate measures of the activation of Akt and Erk, respectively. Lysates were prepared from hippocampus ipsilateral to KA infusion at varying time points $(n=3-7)$ following termination of 40 min KA-SE by treatment with diazepam and separated by SDS-PAGE. Representative Western blots were probed with indicated antibodies. Immunoblotting (IB) B, Quantitative analysis of KA-SE induced the activation of Akt. The immunoreactivity of p-Akt was quantified and normalized over Akt. The data were shown as percentage of the control (Con). C, Quantitative analysis of KA-SE induced the activation of Erk. The immunoreactivity of $p$-Erk was quantified and normalized over Erk1/2. Data are presented as percentage of PBS-infused control and analyzed with one-way ANOVA followed by Dunnett's post hoc test. ${ }^{*} p<0.05,{ }^{* *} p<0.01,{ }^{* * *} p<0.001$.

A

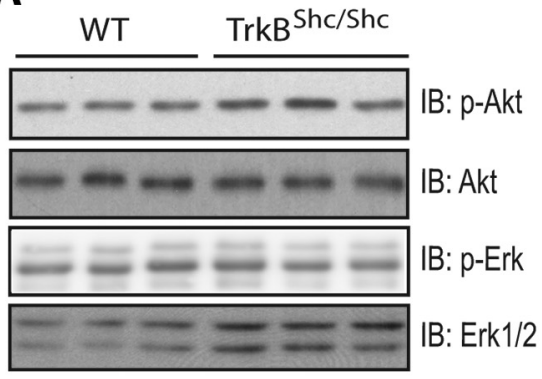

D

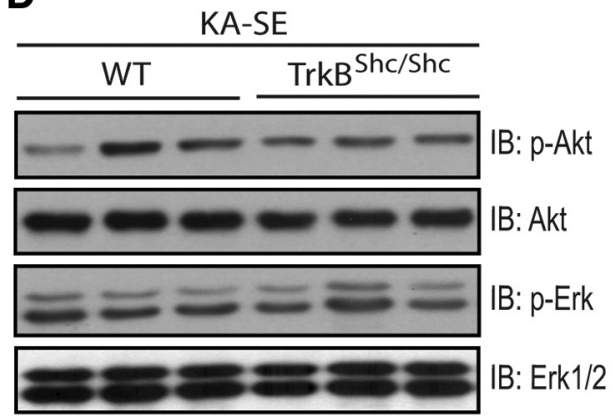

B

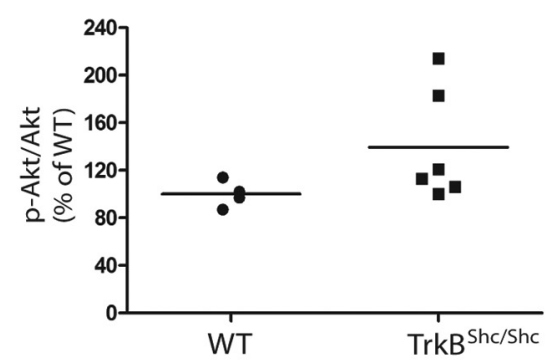

$\mathbf{E}$

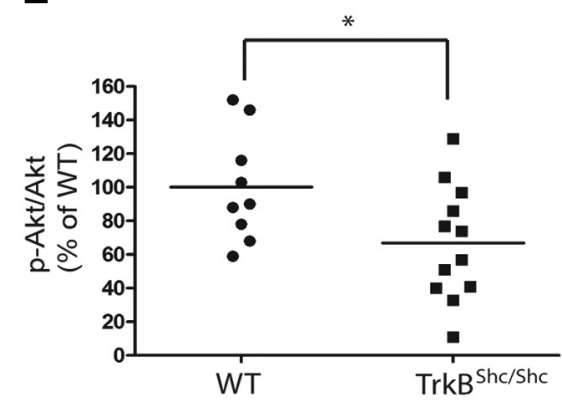

C

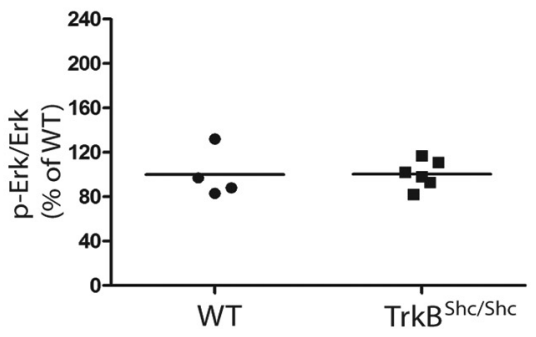

F

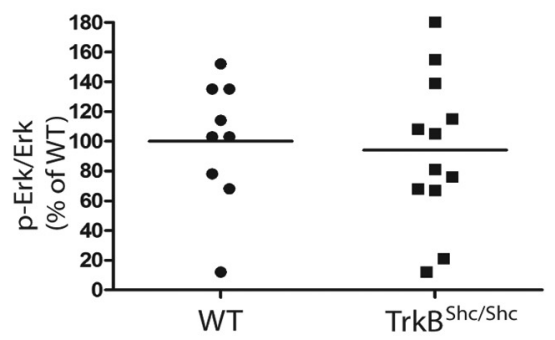

Figure 3. KA-SE induced activation of Akt but not Erk is reduced in TrkB ${ }^{\text {shc/shc }}$ mutant mice. $A$, The level of p-Akt and p-Erk is similar in WT and TrkB ${ }^{\text {shc/shc }}$ mutant mice. Hippocampal lysates were prepared from naive WT $(n=4)$ and $\operatorname{TrkB}{ }^{\text {shc/shc }}(n=6)$ mutant mice and subjected to SDS-PAGE. The blots were probed with indicated antibodies. Immunoblotting (IB) $\boldsymbol{B}$, Quantification of the immunoreactivity of p-Akt over Akt. C, Quantification of the immunoreactivity of p-Erk over Erk. D, KA-SE induced increase of p-Akt was reduced in TrkB ${ }^{\text {shc/shc }}$ mutant mice. Hippocampal lysates (WT: $n=9$; TrkB ${ }^{\text {shc/shc: }} n=12$ ) were prepared at $6 \mathrm{~h}$ following termination of $40 \mathrm{~min}$ SE by treatment with diazepam and separated by SDS-PAGE. Representative Western blots were probed with indicated antibodies. $\boldsymbol{E}, \boldsymbol{F}$, Quantification of $\mathrm{p}$-Akt/Akt and $\mathrm{p}$-Erk/Erk in $\boldsymbol{D}$, respectively. Data are presented as percentage of WT control and analyzed by unpaired Student's $t$ test. ${ }^{*} p<0.05$.

after SE to quantify neuronal degeneration (Mouri et al., 2008; $\mathrm{Gu}$ et al., 2015). Consistent with previous results (Mouri et al., 2008; Gu et al., 2015), SE preferentially induced the death of CA3 pyramidal cells in hippocampus ipsilateral to KA infusion as evident by FJC staining in sections from both WT and TrkB ${ }^{S h c / S h c}$ mice (Fig. 4A). In accord with our hypothesis, striking increases in the number of FJC-positive cells were detected in the CA3 pyramidal cell layer of the middle level of hippocampus in Trk$B^{S h c / S h c}$ relative to WT mice (Fig. $4 B$; WT: $9.1 \pm 3.1$ cells, $n=15$; $\operatorname{Trk} B^{S h c / S h c}: 24.5 \pm 6.0$ cells, $n=11 ; p=0.022$, df $=24$, unpaired $t$ test). We also found increased FJC-positive neurons in sections from rostral hippocampal levels of $\operatorname{Trk} B^{\mathrm{Shc} / S h c}$ mutant mice
$(19.1 \pm 5.2, n=11)$ compared with WT mice $(6.5 \pm 2.1, n=15$, $p=0.020, \mathrm{df}=24$, unpaired $t$ test $)$. Although sections of caudal hippocampus were available only from a subset of the $15 \mathrm{WT}$ animals reported above, the trend of increased numbers of FJClabeled cells in $\operatorname{Trk} B^{S h c / S h c}(28.7 \pm 8.3, n=11)$ compared with WT mice (WT: $10.2 \pm 3.2, n=11, p=0.051$, df $=20$, unpaired $t$ test) persisted. Notably, there was no FJC staining following infusion of vehicle into amygdala in sections from either WT or $\operatorname{Trk} B^{S h c / S h c}$ mice (data not shown). In sum, these analyses reveal increased numbers of FJC-positive cells in hippocampus following SE in $T r k B^{S h c / S h c}$ compared with WT mice, thereby supporting our conclusion that disruption of TrkB-mediated Shc 
A
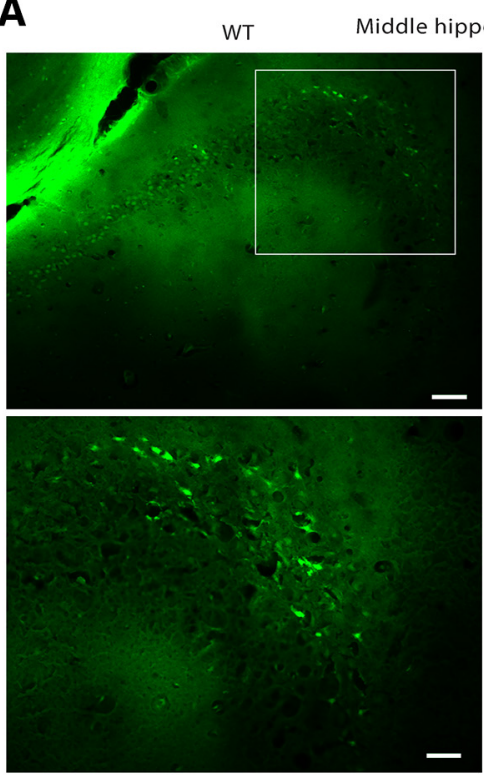

\section{C}

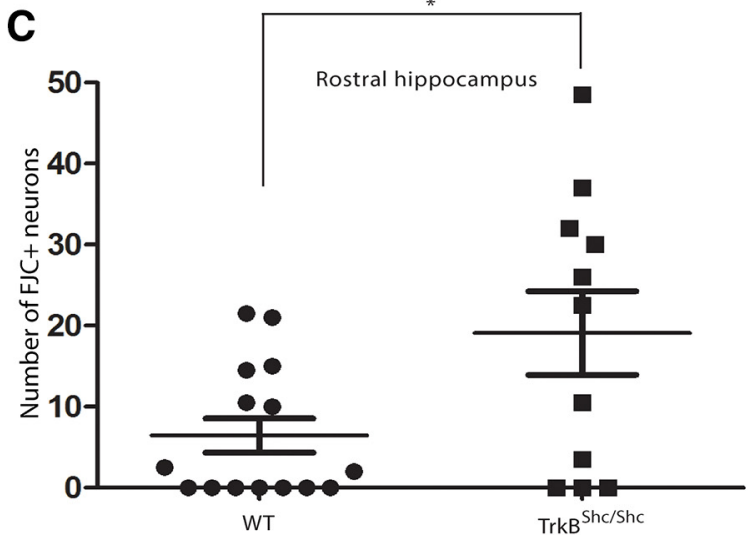

$\operatorname{TrkB}^{\text {Shc/ShC }}$

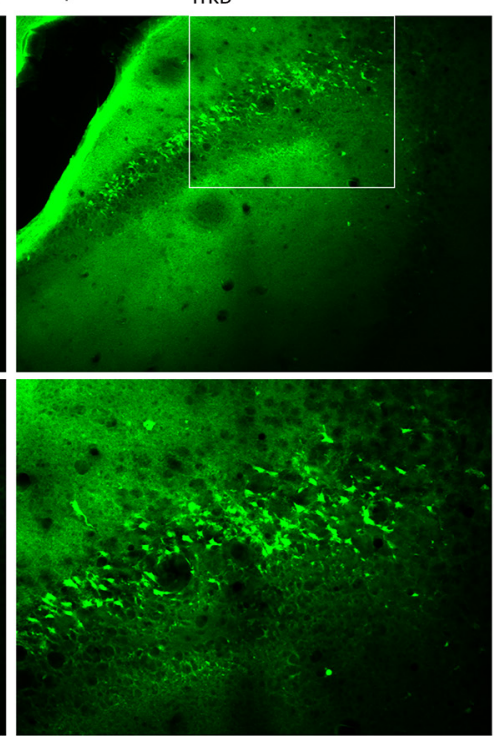

B

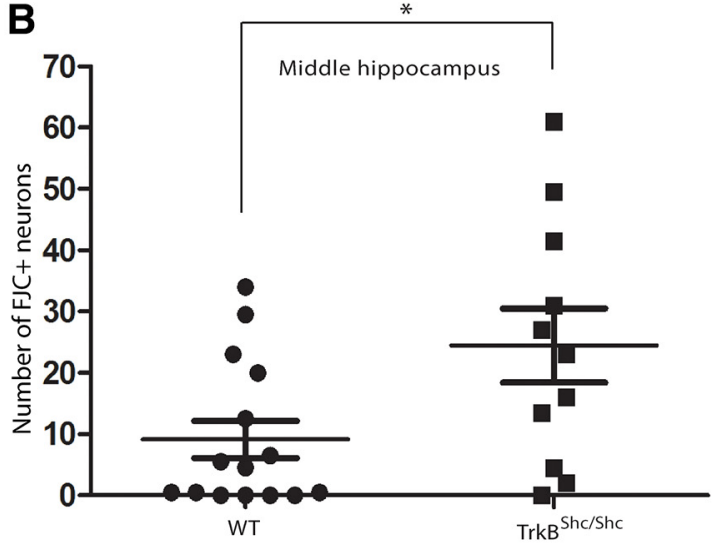

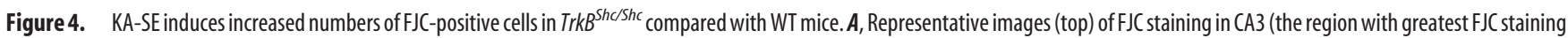
in this model) of middle hippocampus for both WT and TrkB ${ }^{\text {Shc } / S h}$ mice $24 \mathrm{~h}$ after SE. Scale bar, $50 \mu \mathrm{m}$. Insets, High-magnitude images (bottom) of FJC-positive neurons in hippocampal CA3 subfields. Scale bar, $50 \mu \mathrm{m}$. The numbers of FJC-positive cells in CA3 of middle $(\boldsymbol{B})$, rostral $(\boldsymbol{C})$, and caudal (D) hippocampus was quantified. Significantly more FJC-positive cells were found in $T r k B$ shc/shc $(n=$ 11) versus WT $(n=15)$ mice. Data are presented as mean \pm SEM and analyzed by unpaired Student's $t$ test. ${ }^{*} p<0.05$.

signaling enhances hippocampal neuronal cell death following an insult (SE) but not under basal conditions.

\section{Discussion}

We tested the hypothesis that SE-induced activation of TrkB-Shc signaling activates the adaptor protein Akt and mediates neuroprotection following a prolonged seizure episode. We used a genetic strategy, taking advantage of a mouse $\left(\operatorname{TrkB} B^{S h c / S h c}\right)$ containing a point mutation of TrkB preventing the binding of Shc to activated TrkB kinase (Minichiello et al., 1998). Four principal findings emerged: (1) genetic disruption of TrkB-Shc signaling has no effect on behavioral or electrographic measures of SE evoked by IAK infusion; (2) SE induces activation of the prosurvival adaptor protein Akt; (3) SE-induced activation of Akt is dependent in part on TrkB-Shc signaling; and (4) preventing the SE-induced activation of the TrkB-Shc-Akt signaling pathway exacerbated hippocampal neuronal death in vivo after a prolonged seizure episode. Our results are consistent with a model whereby SE-induced activation of TrkB in turn activates the ShcAkt signaling pathway, one consequence of which is limiting SEinduced neurodegeneration.

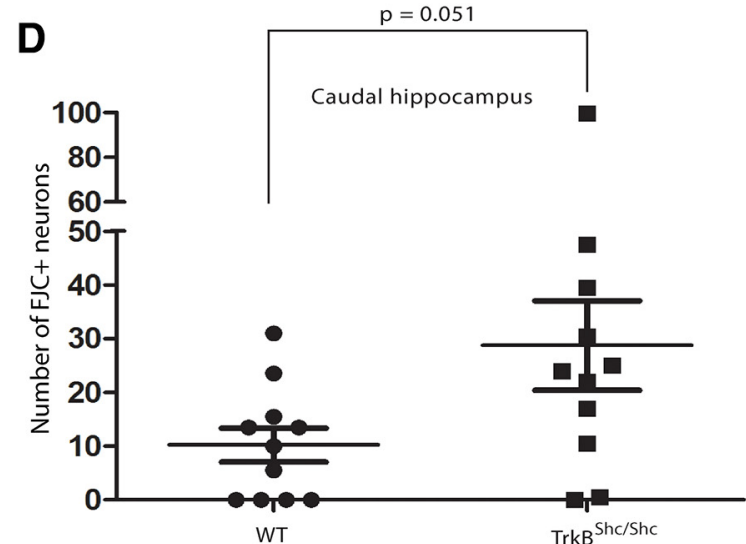

Our study demonstrates that the intensity of behavioral and electrographic seizures of SE is not affected by disrupting TrkBShc signaling (Fig. 1). This is in striking contrast to similar studies using $\operatorname{Trk} B^{P L C / P L C}$ mice, where substituting phenylalanine for tyrosine residue 816 disrupts TrkB-mediated PLC $\gamma 1$ activation (Minichiello et al., 2002). TrkB ${ }^{P L C / P L C}$ mice exhibited a markedly reduced severity of electrographic and behavioral seizures evoked by IAK infusion (Gu et al., 2015). The present findings are consistent with data obtained in the kindling model of epileptogenesis (Goddard et al., 1969) in that development of kindling was markedly impaired in $\operatorname{TrkB} B^{P L C / P L C}$ mice (He et al., 2010) but was indistinguishable from controls in $\operatorname{Trk} B^{S h c / S h c}$ mice (He et al., 2002). Left unanswered are the molecular and biological consequences of TrkB-Shc signaling induced by SE.

To assess the molecular consequences of TrkB-Shc signaling pathways activated by SE, we examined the phosphorylation of Akt and Erk, surrogate measures of activation of PI3K-Akt and MAPK pathways, respectively. We found distinct temporal patterns of SE-induced increases of p-Akt and p-Erk, confirming and extending previous findings (Berkeley et al., 2002; Henshall 
et al., 2002; Kang et al., 2004). SE-induced activation of Erk signaling was similar in WT and TrkB ${ }^{S h c / S h c}$ mice. By contrast, SE-induced p-Akt was partially reduced in $\operatorname{TrkB}{ }^{S h c / S h c}$ mice, indicating that SE induces the activation of Akt in part through TrkBShc signaling. The dependence of Akt but not Erk on TrkB implies that both TrkB-dependent and independent signaling pathways are activated following SE; differences in the time course of activation of these upstream pathways likely accounts for the striking differences in time course of activation of Erk and Akt. Interestingly, the time course of activation of distinct TrkBdependent pathways also differs. In contrast to the peak of Akt activation at $24 \mathrm{~h}$ following status epilepticus observed here (Fig. $2 B$ ), activation of PLC $\gamma 1$ peaked at $6-12 \mathrm{~h}$ and was almost back to baseline by $24 \mathrm{~h}$ (He et al., 2010). Multiple factors could account for the divergent time courses of TrkB-dependent activation of Akt and PLC $\gamma 1$. Analyses of TrkA expressed in a cell line in vitro reveal differences in the temporal pattern of phosphorylation of individual TrkA tyrosines following its activation by NGF; the time course of signaling pathways initiated from these tyrosines would be expected to differ as well (Segal et al., 1996). Importantly, TrkB can undergo activation not only by its prototypical ligand BDNF but also by non-neurotrophin ligands, including the divalent cation zinc in a BDNF independent manner (Lee and Chao, 2001; Reichardt, 2006; Huang et al., 2008); differential activation of TrkB by these distinct stimuli that are dynamically regulated during SE could contribute to divergent time courses of p-Akt and p-PLC $\gamma 1$.

The well documented neuroprotective role of Akt both in vitro (Kim et al., 2002) and in vivo (Henshall et al., 2002) following excitotoxic insults supports the idea that SE-induced TrkB-ShcAkt signaling promotes neuronal survival following SE. These findings in turn are consistent with evidence implicating BDNF in neuronal survival. In vitro studies revealed prosurvival effects of BDNF for both peripheral sensory neurons (Barde et al., 1982) and CNS-derived neurons (Johnson et al., 1986). Genetic deletion of BDNF resulted in loss of immature medium spiny neurons in striatum (Baydyuk et al., 2013). Moreover, BDNF exerts neuroprotective effects following glutamate-induced excitotoxicity in vitro (Cheng and Mattson, 1994; Almeida et al., 2005), a contributor to neuronal death induced by prolonged seizures (Meldrum, 1993). Administration of exogenous BDNF protects against neuronal injury following hypoxic-ischemic injury in vivo (Han et al., 2000). The requirement of TrkB-Shc signaling in BDNF-mediated neuronal survival was demonstrated for mammalian neurons in vitro (Minichiello et al., 1998; Atwal et al., 2000) and for peripheral sensory neurons in vivo in $\operatorname{TrkB}{ }^{S h c / S h c}$ mouse (Minichiello et al., 1998). In contrast to peripheral sensory neurons, both development and survival of CNS neurons was similar in TrkB ${ }^{S h c / S h c}$ and WT control mice; because loss of neocortical CNS neurons is evident in TrkB knock-out mice (Alcántara et al., 1997; Xu et al., 2000), their survival in TrkB ${ }^{\text {Shc/Shc }}$ mice suggests that the activation of Akt by other TrkB-independent signaling pathways could compensate for the impairment of TrkB-Shc signaling. Consistent with this idea, we found that the level of p-Akt was similar in naive WT and $\operatorname{Trk} B^{S h c / S h c}$ mice and revealed impairment only following SE (Fig. $3 A, B$ ). Our present study demonstrates that these non-TrkB-Shc pathways fail to fully compensate in the context of a neuronal deathinducing insult like SE.

What are the cellular and molecular mechanisms by which SE-induced activation of TrkB-Shc-Akt signaling ameliorates neuronal death? Previous work in the IAK model demonstrated that neuronal death is mediated primarily by apoptosis (Araki et al., 2002; Murphy et al., 2007). It is hypothesized that one of the cellular mechanisms by which SE induces neuronal apoptosis is endoplasmic reticulum (ER) stress. Consistent with this idea, application of KA to primary neuronal cultures results in a rapid, substantial disruption of the ER membrane (Sokka et al., 2007; Murphy et al., 2008); furthermore, both the ER stressor tunicamycin and SE produce similar changes in proteins involved in the ER stress response in vivo (Torres-Peraza et al., 2013). Notably, transgenic overexpression of the molecular chaperone 14-3-3 $\zeta$ in mouse brain protects against hippocampal neuronal death following both tunicamycin-induced ER stress and SE in vivo (Brennan et al., 2013), supporting the idea that ER stress contributes to SE-induced neuronal death. Exogenous BDNF prevents tunicamycin-induced neuronal death in primary cultures of cortical neurons through the activation of Akt (Shimoke et al., 2004). It thus seems plausible that one mechanism underlying the neuroprotective effects of TrkB-Shc-Akt signaling is by reducing the severity of seizure-induced ER stress.

The present findings provide additional evidence of distinct biological consequences of individual signaling pathways downstream of TrkB following its activation by SE in vivo. In contrast to the epileptogenic and anxiogenic consequences of SE-induced TrkB-PLC $\gamma 1$ signaling (Gu et al., 2015), the present work reveals neuroprotective consequences of SE-induced TrkB-Shc signaling. Linking the beneficial neuroprotective consequences of SEinduced TrkB activation to TrkB-Shc signaling in particular strengthens the rationale for therapeutic strategies that intervene downstream of TrkB itself. In contrast to targeting TrkB kinase, which inhibits all downstream signaling, "selective antagonism" of individual signaling pathways by peptides or small molecules can disrupt a pathway with deleterious consequences while leaving those with beneficial consequences intact. The molecular organization of receptor tyrosine kinases whereby motifs of their cytoplasmic domain interact with $\mathrm{SH} 2$ or PTB domains of signaling adaptor proteins provides defined protein-protein interactions that can be targeted for development of selective peptide or small molecule inhibitors. A detailed understanding of the contribution of individual signaling pathways to the diverse biological consequences of activation of a receptor tyrosine kinase such as TrkB in the context of disease is critical to the rationale for this strategy. Knowledge that the activation of TrkB-Shc-Akt signaling contributes to the beneficial neuroprotective consequences following SE strengthens the rationale for selectively disrupting the TrkB-PLC $\gamma 1$ pathway for limiting the anxiogenic and epileptogenic consequences of SE.

\section{References}

Alcántara S, Frisén J, del Río JA, Soriano E, Barbacid M, Silos-Santiago I (1997) TrkB signaling is required for postnatal survival of CNS neurons and protects hippocampal and motor neurons from axotomy-induced cell death. J Neurosci 17:3623-3633.

Almeida RD, Manadas BJ, Melo CV, Gomes JR, Mendes CS, Graos MM, Carvalho RF, Carvalho AP, Duarte CB (2005) Neuroprotection by BDNF against glutamate-induced apoptotic cell death is mediated by ERK and PI3-kinase pathways. Cell Death Diff 12:1329.

Annegers JF, Hauser WA, Shirts SB, Kurland LT (1987) Factors prognostic of unprovoked seizures after febrile convulsions. N Engl J Med 316:493498.

Araki T, Simon RP, Taki W, Lan JQ, Henshall DC (2002) Characterization of neuronal death induced by focally evoked limbic seizures in the C57BL/6 mouse. J Neurosci Res 69:614-621.

Atwal JK, Massie B, Miller FD, Kaplan DR (2000) The TrkB-shc site signals neuronal survival and local axon growth via MEK and PI3-kinase. Neuron 27:265-277.

Barde YA, Edgar D, Thoenen H (1982) Purification of a new neurotrophic factor from mammalian brain. EMBO J 1:549-553. 
Baydyuk M, Xie Y, Tessarollo L, Xu B (2013) Midbrain-derived neurotrophins support survival of immature striatal projection neurons. J Neurosci 33:3363-3369.

Berkeley JL, Decker MJ, Levey AI (2002) The role of muscarinic acetylcholine receptor-mediated activation of extracellular signal-regulated kinase 1/2 in pilocarpine-induced seizures. J Neurochem 82:192-201.

Borges K, Gearing M, McDermott DL, Smith AB, Almonte AG, Wainer BH, Dingledine R (2003) Neuronal and glial pathological changes during epileptogenesis in the mouse pilocarpine model. Exp Neurol 182:21-34.

Brennan GP, Jimenez-Mateos EM, McKiernan RC, Engel T, Tzivion G, Henshall DC (2013) Transgenic overexpression of 14-3-3 zeta protects hippocampus against endoplasmic reticulum stress and status epilepticus in vivo. PloS One 8:e54491.

Cheng B, Mattson MP (1994) NT-3 and BDNF protect CNS neurons against metabolic/excitotoxic insults. Brain Res 640:56-67.

French JA (2007) Refractory epilepsy: clinical overview. Epilepsia 48:3-7.

French JA, Williamson PD, Thadani VM, Darcey TM, Mattson RH, Spencer SS, Spencer DD (1993) Characteristics of medial temporal lobe epilepsy: I. Results of history and physical examination. Ann Neurol 34:774-780.

Goddard GV, McIntyre DC, Leech CK (1969) A permanent change in brain function resulting from daily electrical stimulation. Exp Neurol 25:295330.

Gu B, Huang YZ, He XP, Joshi RB, Jang W, McNamara JO (2015) A peptide uncoupling BDNF receptor TrkB from phospholipase $\mathrm{C} \gamma 1$ prevents epilepsy induced by status epilepticus. Neuron 88:484-491.

Han BH, D'Costa A, Back SA, Parsadanian M, Patel S, Shah AR, Gidday JM, Srinivasan A, Deshmukh M, Holtzman DM (2000) BDNF blocks caspase-3 activation in neonatal hypoxia-ischemia. Neurobiol Dis 7: $38-53$.

He XP, Minichiello L, Klein R, McNamara JO (2002) Immunohistochemical evidence of seizure-induced activation of trkB receptors in the mossy fiber pathway of adult mouse hippocampus. J Neurosci 22:7502-7508.

He XP, Pan E, Sciarretta C, Minichiello L, McNamara JO (2010) Disruption of TrkB-mediated phospholipase $\mathrm{C} \gamma$ signaling inhibits limbic epileptogenesis. J Neurosci 30:6188-6196.

Henshall DC, Araki T, Schindler CK, Lan JQ, Tiekoter KL, Taki W, Simon RP (2002) Activation of bcl-2-associated death protein and counterresponse of Akt within cell populations during seizure-induced neuronal death. J Neurosci 22:8458-8465.

Hesdorffer DC, Shinnar S, Lax DN, Pellock JM, Nordli DR Jr, Seinfeld S, Gallentine W, Frank LM, Lewis DV, Shinnar RC, Bello JA, Chan S, Epstein LG, Moshé SL, Liu B, Sun S (2016) Risk factors for subsequent febrile seizures in the FEBSTAT study. Epilepsia 57:1042-1047.

Huang YZ, Pan E, Xiong ZQ, McNamara JO (2008) Zinc-mediated transactivation of TrkB potentiates the hippocampal mossy fiber-CA3 pyramid synapse. Neuron 57:546-558.

Johnson JE, Barde YA, Schwab M, Thoenen H (1986) Brain-derived neurotrophic factor supports the survival of cultured rat retinal ganglion cells. J Neurosci 6:3031-3038.

Kang UG, Roh MS, Jung JR, Shin SY, Lee YH, Park JB, Kim YS (2004) Activation of protein kinase B (Akt) signaling after electroconvulsive shock in the rat hippocampus. Progr Neuro-Psychopharmacol Biol Psychiatry 28:41-44.

Kim AH, Yano H, Cho H, Meyer D, Monks B, Margolis B, Birnbaum MJ, Chao MV (2002) Aktl regulates a JNK scaffold during excitotoxic apoptosis. Neuron 35:697-709.

Lee FS, Chao MV (2001) Activation of Trk neurotrophin receptors in the absence of neurotrophins. Proc Natl Acad Sci U S A 98:3555-3560.

Lehmkuhle MJ, Thomson KE, Scheerlinck P, Pouliot W, Greger B, Dudek FE (2009) A simple quantitative method for analyzing electrographic status epilepticus in rats. J Neurophysiol 101:1660-1670.

Liu G, Gu B, He XP, Joshi RB, Wackerle HD, Rodriguiz RM, Wetsel WC, McNamara JO (2013) Transient inhibition of TrkB kinase after status epilepticus prevents development of temporal lobe epilepsy. Neuron 79:31-38.

McNamara JO, Scharfman HE (2012) Temporal lobe epilepsy and the BDNF receptor, TrkB. In: Jasper's basic mechanisms of the epilepsies [Internet], Ed 4 (Noebels JL, Avoli M, Rogawski MA, Olsen RW, DelgadoEscueta AV, eds). Bethesda, MD: National Center for Biotechnology Information.

Meldrum BS (1993) Excitotoxicity and selective neuronal loss in epilepsy. Brain Pathol 3:405-412.

Minichiello L, Casagranda F, Tatche RS, Stucky CL, Postigo A, Lewin GR, Davies AM, Klein R (1998) Point mutation in trkB causes loss of NT4dependent neurons without major effects on diverse BDNF responses. Neuron 21:335-345.

Minichiello L, Calella AM, Medina DL, Bonhoeffer T, Klein R, Korte M (2002) Mechanism of TrkB-mediated hippocampal long-term potentiation. Neuron 36:121-137.

Mouri G, Jimenez-Mateos E, Engel T, Dunleavy M, Hatazaki S, Paucard A, Matsushima S, Taki W, Henshall DC (2008) Unilateral hippocampal CA3-predominant damage and short latency epileptogenesis after intraamygdala microinjection of kainic acid in mice. Brain Res 1213:140-151.

Murphy B, Dunleavy M, Shinoda S, Schindler C, Meller R, Bellver-Estelles C, Hatazaki S, Dicker P, Yamamoto A, Koegel I, Chu X, Wang W, Xiong Z, Prehn J, Simon R, Henshall D (2007) Bcl-w protects hippocampus during experimental status epilepticus. Am J Pathol 171:1258-1268.

Murphy N, Bonner HP, Ward MW, Murphy BM, Prehn JH, Henshall DC (2008) Depletion of 14-3-3 zeta elicits endoplasmic reticulum stress and cell death, and increases vulnerability to kainate-induced injury in mouse hippocampal cultures. J Neurochem 106:978-988.

Pitkänen A (2010) Therapeutic approaches to epileptogenesis: hope on the horizon. Epilepsia 51:2-17.

Racine RJ (1972) Modification of seizure activity by electrical stimulation: II. Motor seizure. Electroencephalogr Clin Neurophysiol 32:281-294.

Reichardt LF (2006) Neurotrophin-regulated signalling pathways. Philos Trans R Soc Lond B Biol Sci 361:1545-1564.

Segal RA, Bhattacharyya A, Rua LA, Alberta JA, Stephens RM, Kaplan DR, Stiles CD (1996) Differential utilization of Trk autophosphorylation sites. J Biol Chem 271:20175-20181.

Shimoke K, Utsumi T, Kishi S, Nishimura M, Sasaya H, Kudo M, Ikeuchi T (2004) Prevention of endoplasmic reticulum stress-induced cell death by brain-derived neurotrophic factor in cultured cerebral cortical neurons. Brain Res 1028:105-111.

Sokka AL, Putkonen N, Mudo G, Pryazhnikov E, Reijonen S, Khiroug L, Belluardo N, Lindholm D, Korhonen L (2007) Endoplasmic reticulum stress inhibition protects against excitotoxic neuronal injury in the rat brain. J Neurosci 27:901-908.

Torres-Peraza JF, Engel T, Martín-Ibáñez R, Sanz-Rodríguez A, FernándezFernández MR, Esgleas M, Canals JM, Henshall DC, Lucas JJ (2013) Protective neuronal induction of ATF5 in endoplasmic reticulum stress induced by status epilepticus. Brain 136:1161-1176.

Treiman DM, Walton NY, Kendrick C (1990) A progressive sequence of electroencephalographic changes during generalized convulsive status epilepticus. Epilepsy Res 5:49-60.

Tsai MH, Chuang YC, Chang HW, Chang WN, Lai SL, Huang CR, Tsai NW, Wang HC, Lin YJ, Lu CH (2009) Factors predictive of outcome in patients with de novo status epilepticus. QJM 102:57-62.

Walton NY, Treiman DM (1988) Response of status epilepticus induced by lithium and pilocarpine to treatment with diazepam. Exp Neurol 101: 267-275.

Xu B, Zang K, Ruff NL, Zhang YA, McConnell SK, Stryker MP, Reichardt LF (2000) Cortical degeneration in the absence of neurotrophin signaling: dendritic retraction and neuronal loss after removal of the receptor TrkB. Neuron 26:233-245. 